\title{
Accumulation of organochlorinated pesticides by triolein-containing semipermeable membrane device (triolein-SPMD) and rainbow trout
}

\author{
Yibing Lu, Zijian Wang* \\ State Key Laboratory of Environmental Aquatic Chemistry, Research Center for Eco-Environmental Sciences, \\ Chinese Academy of Sciences, Beijing 100085, China
}

Received 15 February 2002; received in revised form 24 September 2002; accepted 18 November 2002

\begin{abstract}
An important advance in aquatic ecotoxicology is the development and application of biomimetic sampling technology for hydrophobic contaminants. In this paper, accumulation kinetics of five organochlorinated pesticides, i.e. hexachlorobenzene, lindan, aldrin, heptachlor epoxide and 4,4'-DDT, by triolein-containing semipermeable membrane device (triolein-SPMD) and by fish (rainbow trout) were compared in a laboratory continuous flow system. Accumulation kinetics of organochlorinated pesticides by the triolein-SPMD and by rainbow trout were linear during the exposure period of $20 \mathrm{~d}$, except for lindan. Approach of an asymptote could be observed for lindan at the end of the exposure for rainbow trout. When the kinetic data were used to calculate the first-order uptake rate constants, it was found that the uptake rates of the chemicals in triolein-SPMD were 1-2.5 times higher than those in the fish. The estimated bioconcentration factors of the five pesticides in rainbow trout were in the range of 1000-7000 and the device concentration factor in the range of 1500-18 000. Although equilibrium was not reached for most pesticides under 21-d exposure period, correlations between $C_{\mathrm{L}} / C_{\mathrm{w}}$ and $K_{\mathrm{ow}}\left(R^{2}=0.887\right)$ and $C_{\mathrm{f}} / C_{\mathrm{w}}$ and $K_{\mathrm{ow}}\left(R^{2}=0.931\right)$ could be observed which was a clear indication that triolein-SPMD accumulates chlorinated pesticides in a quite similar way as fish do. Our results show that triolein-SPMD could serve as a good surrogate for rainbow trout for simulating accumulations of chlorinated pesticides and may be used as a universal surrogate for fish in natural waters.
\end{abstract}

(C) 2003 Elsevier Science Ltd. All rights reserved.

Keywords: Organochlorinated pesticides; Accumulations; Triolein semi-permeable membrane devices; Rainbow trout

\section{Introduction}

There is a clear need for rapid, easy to use, effective and low-cost integrative methods that allow not only the direct monitoring of the fate and concentrations of trace level of chemical-contaminant mixture in the environment, but also assessment of their hazards to aquatic organisms and human health. Membrane-based passive samplers seem to be a promising tool for the timeintegrated monitoring of hydrophobic pollutants in

\footnotetext{
*Corresponding author. Tel.: + 86-10-628-49-1-40; fax: + 8610-629-23-5-43.

E-mail address: wangzj@mail.rcees.ac.cn (Z. Wang).
}

aquatic ecosystems. In these devices the uptake of chemicals is based on the process of passive partitioning of a compound between water and a lipophilic solvent enclosed in a semipermeable polymeric membrane. Thus, the passive samplers can be used as indicators of bioavailability of chemical pollutants. Furthermore, in contrast to most living organisms, they can be exposed to harsh environmental conditions for long time periods and still remain operative [1]. Among the several designs of membrane samplers that have been used, trioleincontaining semi-permeable membrane devices (trioleinSPMD), proposed by Huckins et al. [2], consisting of a thin film of neutral lipid, enclosed in thin-walled layflat tubing, made of low-density polyethylene, proved to be 
most effective in their capacity to accumulate lipophilic substances. The molecular size-exclusion limit of the polyethylene membrane is similar to that of biological membranes while triolein, usually used in trioleinSPMD, constitutes a significant fraction of the lipid pool of most aquatic organisms. Triolein-SPMD has received wide recognition as an effective tool for environmental research and analysis. It has been successfully used for monitoring chemicals in aquatic environments [2-8], and in air $[9,10]$. Triolein-SPMD has also been used for screening and preliminary identification of emission sources in wastewater systems and groundwater [11-14], and appears to simulate the bioconcentration of dissolved chemicals by aquatic organisms [15-21]. The procedure for dialytic recovery of pesticides can be used separately to remove lipids and other interferences from environmental sample extracts [22,23].

Studies have been carried out on the comparisons of accumulations of a variety of organic contaminants by triolein-SPMD and by several types of aquatic organisms, but different results were obtained. In the present work, Five chlorinated pesticides were exposed to triolein-SPMD and rainbow trout, which is a model fish for standard tests for chemicals [24]. We report the uptake kinetics and bioconcentration factors (BCFs) of five chlorinated pesticides in rainbow trout and in triolein-SPMD and the similarity and differences between the two sampling approaches to examine how well triolein-SPMD mimics rainbow trout in terms of discriminatory uptake of hydrophobic chemicals and further explore the possibility of using triolein-SPMD to concentrate bioavailable environmental contaminants.

\section{Materials and methods}

\subsection{Test chemicals and reagents}

The pesticides-grade lindan $(99.0 \%$ purity), heptachlor epoxide $(99.0 \%$ purity), aldrin $(99.9 \%$ purity) and hexachlorobenzene ( $99.0 \%$ purity) were purchased from the Institute of Organic Industrial Chemistry (Warsaw, Poland). 4,4'-DDT (99\% purity) was purchased from Sigma Chemical Company (St. Louis, MO, USA). Organic solvents employed in the experiment, such as cyclohexane $\left(\mathrm{C}_{6} \mathrm{H}_{12}\right)$, petroleum ether (boiling point range is $\left.60-90^{\circ} \mathrm{C}\right)$, acetone $\left(\mathrm{CH}_{3} \mathrm{COCH}_{3}\right)$, analytical grade ethanol (Beijing Chem. Co.) were distilled by allglass systems before use.

\subsection{Semipermeable membrane devices}

Triolein-SPMD was prepared in a similar way as described by Huckins et al. [2]. Layflat polyethylene tubing (width $3.0 \mathrm{~cm}$, membrane thickness ranged from 50 to $55 \mu \mathrm{m}$, from Beijing Plastics company) was cut into pieces of $23 \mathrm{~cm}$ in length, soaked in cyclohexane for $24 \mathrm{~h}$ to remove trace contaminants, and dried with ultra-pure nitrogen. The total mass of tubing was $1.45 \mathrm{~g}$, and the ratio of mass of tubing to volume of solvent was $0.01 \mathrm{~g} /$ $\mathrm{ml}$. Then $250 \mu \mathrm{l}(227.5 \mathrm{mg})$ of triolein was coated onto the polyethylene tubing before the ends were sealed with a plastic sealer (Model SF-300, China). The ratio of triolein-SPMD surface area to triolein volume is $400 \mathrm{~cm}^{2} / \mathrm{ml}$. The tube was formed into twisted loops and glass sticks were used as anchors to keep the device in a vertical position when suspended in the test solution.

\subsection{Rainbow trout}

The rainbow trout were obtained from Shuntong Aquatic Institute in Huairou, Beijing. The average length and weight of the fish were $9.4 \pm 0.6 \mathrm{~cm}$ and $8.8 \pm 1.5 \mathrm{~g} \quad(n=20)$, respectively. The average lipid content calculated on wet weight was $10.0 \pm 2.1 \%$. The fish were sterilized and reared under laboratory condition for 2 weeks before experiment. The death rate during the domestication was lower than $2 \%$.

\subsection{Exposure experiment}

The experiment was conducted in a glass exposure chamber (volume 701 ). A set of 21 triolein-SPMDs and 25 fish was placed in the exposure chamber, which was connected to a continuous-flow system. The flow rate through the chamber was maintained at about $300 \mathrm{ml} /$ min (the flow rate through the test chambers was 5.76 volume additions per $24 \mathrm{~h}$ ). Concentrated solution of the five pesticides in ethanol (concentration ratio 1:1:1:1:1) was supplied into the main flow up the exposure chamber by a peristaltic pump. The rate of the peristaltic pump was adjusted to obtain concentrations of the pesticides of about $2 \mu \mathrm{g} / 1$, with a negligible concentration of ethanol (less than $0.1 \mathrm{ml} / \mathrm{l}$ ). The water temperature was maintained at $20^{\circ} \mathrm{C}$. The experiment lasted for $20 \mathrm{~d}$, during which the rainbow trout received no food. On days $0.5,1$, $2,4,8,14$ and 20 , three water samples of each $50 \mathrm{ml}$, three triolein-SPMDs and three fish were taken and processed to determine concentrations of the pesticides. The rainbow trout were stored in glass jars at $-20^{\circ} \mathrm{C}$ until analysis. Control triolein-SPMD (three devices) and rainbow trout (three individuals) were taken at the beginning of the experiment, just before the connection of the exposure chamber to the continuous-flow system.

\section{Analytical methods}

\subsection{Water}

The pesticides were spiked with known amounts of surrogate standard before extraction with $20 \mathrm{ml}$ aliquots 
of petroleum ether. The extract was reduced in volume to $0.5 \mathrm{ml}$ using nitrogen blow-down technique. Aliquots of the extract were spiked with the instrumental internal standard and analyzed using a gas chromatograph (HP5890 Series II) equipped with a ${ }^{63} \mathrm{Ni}$ electron capture detector (GC-ECD) and a HP-5 fused silica capillary column $(30 \mathrm{~m} \times 0.32 \mathrm{~mm}$ i.d., film thickness of $0.17 \mu \mathrm{m})$. The temperatures of the injector and of the detector were kept at $250^{\circ} \mathrm{C}$ and $260^{\circ} \mathrm{C}$, respectively. Column temperature was programmed at $85^{\circ} \mathrm{C}$ increasing at $10^{\circ} \mathrm{C} / \mathrm{min}$ to $180^{\circ} \mathrm{C}$, and then increasing at $20^{\circ} \mathrm{C} / \mathrm{min}$ to $280^{\circ} \mathrm{C}$, and a held for $30 \mathrm{~min}$ at $280^{\circ} \mathrm{C}$. Nitrogen gas of extra purity was used as a carrier gas. The flow rate was $1 \mathrm{ml} / \mathrm{min}$ and the pressure was kept at 20 psi.

\subsection{Semipermeable membrane devices}

After sampling, triolein-SPMD was first rinsed under running tap water. After soft papers drying, they were transferred into glass jars with ground edge caps. Cyclohexane $(50 \mathrm{ml})$ was added, and the membranes were dialyzed for $48 \mathrm{~h}$ at $20^{\circ} \mathrm{C}$. Five milliliter of the dialysates was taken up and was reduced to about $2 \mathrm{ml}$ using rotary evaporation and then reduced to $1 \mathrm{ml}$ by applying a gentle stream of high-pure nitrogen gas. GCECD was used for analysis at the same conditions as analysis of water extracts.

\subsection{Rainbow trout}

The rainbow trout were thawed at room temperature for several hours. After length and wet weight determination, each fish was sawed into small pieces, ground with a meat grinder, and then blended with anhydrous $\mathrm{Na}_{2} \mathrm{SO}_{4}$ (baked at $475^{\circ} \mathrm{C}$ prior to use). The mixture was packed into Soxhlet extractor and then was extracted with mixture of cyclohexane, acetone and petroleum ether $(1: 1: 1=\mathrm{v}: \mathrm{v}: \mathrm{v})$ for $24 \mathrm{~h}$. The extract was divided into two parts. One part $(50 \%$ of the total) was concentrated by rotation evaporator to about $1 \mathrm{ml}$ then was passed through a silica gel column (60-80 mesh, activated at $180 \pm 2^{\circ} \mathrm{C}$ for $12 \mathrm{~h}$ before use) and then a glass-column (10 mm I. D) filled with $12 \mathrm{~cm}$ of silica gel. The column was eluted with $50 \mathrm{ml}$ cyclohexane. After concentrating to a final volume of $1 \mathrm{ml}$ using a gentle stream of extra purity nitrogen and adding in the internal standards, $1 \mu \mathrm{l}$ of the purified extract was injected into GC/ECD in splitless mode to analyze the concentrations of pesticides in the same way as water samples analysis procedure. The other part was used to measure the lipid content, using the method described previously [2]. Analytical procedure was the same as that for water extract.

\section{Quality control}

For quality control of gas chromatographic conditions, a checkout procedure was performed before sample analysis. In the procedure, a standard mixture with DDT content of $15 \%$ was used and the calibration was carried out when the percentage of DDT in standard mixture deviated significantly from $15 \%$.

One triolein-SPMD blank (a freshly prepared trioleinSPMD) and two reagent blanks ( $100 \mathrm{ml}$ of cyclohexane and $100 \mathrm{ml}$ of dichloromethane, blow down to $1 \mathrm{ml}$, respectively) were used for the entire clean up and enrichment procedure. No chemicals of concern were found in these blanks. 2,4,5,6-tetrachloro- $m$-xylene (TMX) and decachlorobiphenyl (PCB209) were used as surrogate and 4,4'-dichlorobiphenyl (PCB15) was used as an internal standard.

To $1 \mathrm{~g}$ of fish or $1 \mathrm{~g}$ of triolein-SPMD, $1 \mu \mathrm{g}$ of each organochlorinated pesticide was spiked before extraction. Three replicates were used. The recoveries of all the pesticides were in the range of $76-102 \%$ with variation coefficients not exceeding $15 \%$. There were no significant differences between spiking levels and recoveries, and the concentration estimates were not corrected for these recoveries.

\section{Results and discussion}

\subsection{Uptake kinetics}

Properties of the five pesticides are shown in Table 1. The time-dependent bioconcentration processes of

Table 1

Selected physicochemical properties of the test organochlorinated pesticides and their concentrations in water

\begin{tabular}{lllll}
\hline Compound & Molecular weight & Water solubility $\mu \mathrm{g} / 1$ & $\log K_{\text {ow }}{ }^{\mathrm{a}}$ & Concentration in water $(\mu \mathrm{g} / \mathrm{l}, \mathrm{mean}+\mathrm{SD})^{\mathrm{b}}$ \\
\hline Lindan & 291 & 7800 & 3.9 & $2.36 \pm 1.70$ \\
Aldrin & 365 & 180 & 5.3 & $1.31 \pm 0.69$ \\
Heptachlor epoxide & 389.2 & 350 & 5.4 & $1.52 \pm 0.53$ \\
$4,4^{\prime}$-DDT & 354.5 & 5.5 & 5.7 & $1.14 \pm 1.20$ \\
Hexachlorobenzene & 284.8 & - & 6.2 & $1.07 \pm 0.99$ \\
\hline
\end{tabular}

${ }^{\mathrm{a}}$ Cited from [22].

${ }^{\mathrm{b}}$ Mean value of all samples taken over the entire exposure period, 21 samples in total. 

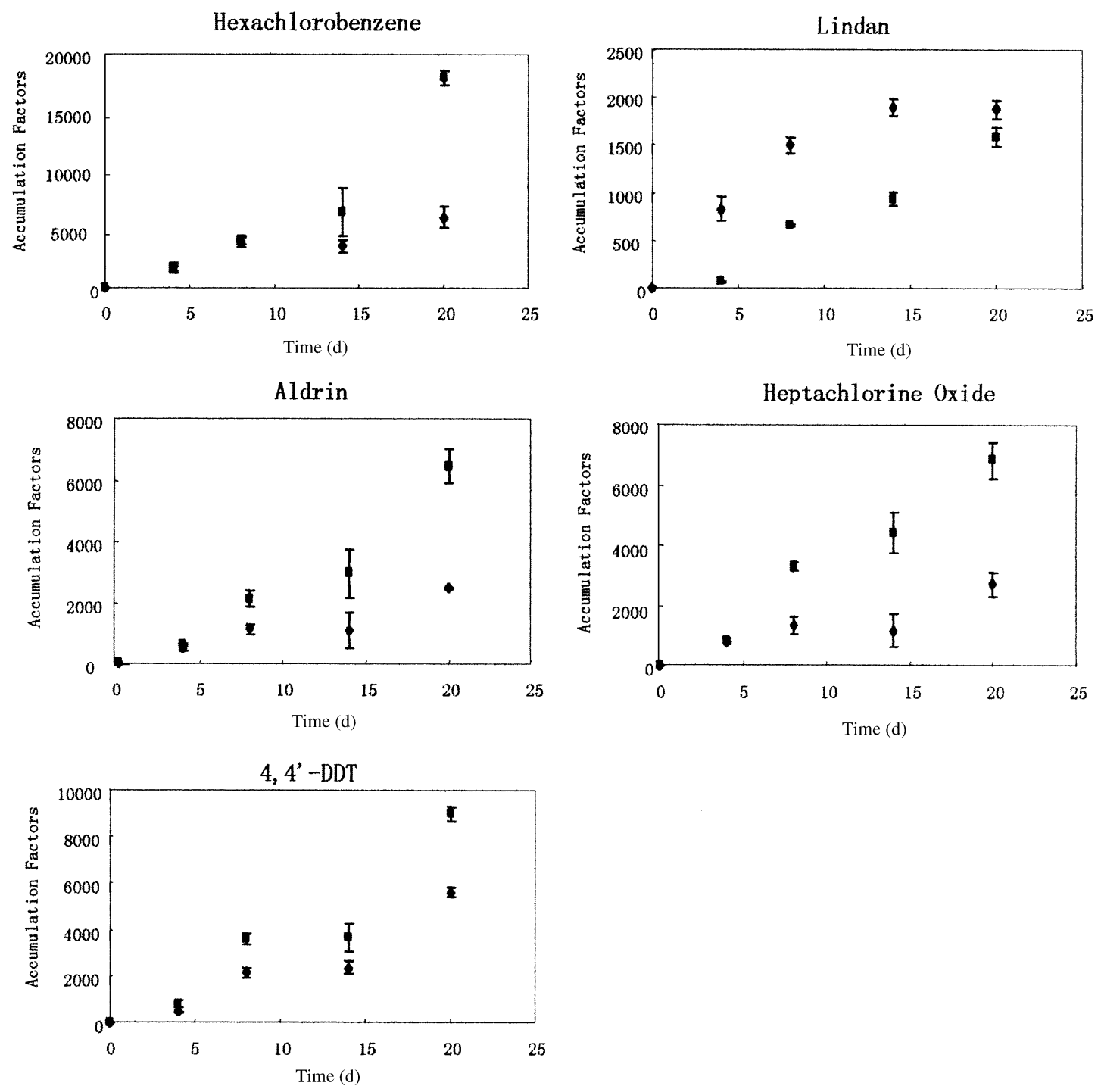

Fig. 1. The uptake kinetics of selected pesticides by triolein-SPMD and rainbow trout, where $(\boldsymbol{\square})$ and $(\bullet)$ indicate triolein-SPMD and rainbow trout, respectively.

pesticides by the triolein-SPMD and by fish are shown in Fig. 1. A quick sequestration, especially at the beginning of the exposure, and a linear increase during the entire exposure period of $20 \mathrm{~d}$ was observed for all tested chemicals, excepting for the bioaccumulation of lindan by the fish (Fig. 1). For lindan in the fish, after $20 \mathrm{~d}$ a steady state of exposure could be observed.

To predict chemical concentration in water by using its concentration in triolein-SPMD, the sampling rate $\left(R_{\mathrm{S}}\right)$ is required, which could be obtained from the linearity of uptake kinetics. To obtain the $R_{\mathrm{s}}$, a mathematical model was used to fit the experimental data. In the model, the concentration of a chemical in the triolein-SPMD, or $C_{\mathrm{L}}$, should be proportional to $R_{\mathrm{S}}$ when it is linearly proportional to the concentration of the chemical in water, or $C_{\mathrm{w}}$, as shown by the following equation:

$C_{\mathrm{L}}=C_{\mathrm{w}} R_{\mathrm{s}} t / V_{\mathrm{L}}$,

where $V_{\mathrm{L}}$ is the volume of the triolein-SPMD and $t$ is the exposure period (in d). By least-squares linear regression for the linear part of the experimental data, a good fit was obtained for all pesticides with $r^{2}$ values from 0.80 to 0.93 for rainbow trout and from 0.79 to 0.95 for triolein-SPMD. Variation coefficients for $R_{\mathrm{S}}$ were less than $50 \%$ for rainbow trout and less than $30 \%$ for triolein-SPMD, respectively (Table 2 ). The uptake rate 
Table 2

Calculated sampling rates of pesticides by rainbow trout and by triolein-SPMD

\begin{tabular}{|c|c|c|c|c|c|c|c|c|}
\hline \multirow[t]{3}{*}{ Compounds } & \multicolumn{4}{|c|}{$R_{\mathrm{s}}($ whole body based $)$} & \multicolumn{4}{|c|}{$R_{\mathrm{S}}$ (lipid based) } \\
\hline & \multicolumn{2}{|c|}{ Rainbow trout } & \multicolumn{2}{|c|}{ SPMDs } & \multicolumn{2}{|c|}{ Rainbow trout } & \multicolumn{2}{|c|}{ SPMDs } \\
\hline & $\mathrm{L} / \mathrm{d}$ & CV $(\%)$ & $\mathrm{L} / \mathrm{d}$ & $\mathrm{CV}(\%)$ & $\mathrm{L} / \mathrm{d}$ & $\mathrm{CV}(\%)$ & $\mathrm{L} / \mathrm{d}$ & CV $(\%)$ \\
\hline Lindan & - & - & 0.09 & 11 & - & - & 0.3 & 12 \\
\hline Aldrin & 0.12 & 28 & 0.34 & 21 & 1.26 & 5 & 1.07 & 20 \\
\hline Heptachlor epoxide & 0.18 & 37 & 0.45 & 14 & 1.69 & 21 & 1.43 & 12 \\
\hline $4,4^{\prime}-\mathrm{DDT}$ & 0.24 & 26 & 0.56 & 1 & 2.61 & 19 & 1.14 & 6 \\
\hline Hexachlorobenzene & 0.28 & 12 & 0.61 & 7 & 3.69 & 18 & 1.94 & 5 \\
\hline
\end{tabular}

of pesticides by rainbow trout was defined as $R_{\mathrm{f}}$, which could be obtained by using Eq. (1) in a similar way.

By definition, $R_{\mathrm{s}}$ is indicative of the potentiality of a chemical entering the triolein-SPMD and $R_{\mathrm{f}}$ is for fish, respectively. Generally, $R_{\mathrm{s}}$ and $R_{\mathrm{f}}$ could be expressed as wet weight base (in case of triolein-SPMD, it includes weights of membrane and triolein). In Table $2, R_{\mathrm{s}}$ 's were 1-2.5 times higher than $R_{\mathrm{f}}$ 's. The rates followed the increasing order of lindan $<$ aldrin $<$ heptachlor epoxide $<4,4^{\prime}$-DDT $<$ hexachlorobenzene. This order is also the order of their lipophilicity or octanol/water partitioning coefficients $\left(K_{\mathrm{ow}}\right)$. Moreover, a lower variability could be noted in the case of triolein-SPMD, because triolein-SPMD was a reproducible standardized design, while individual discrimination of fish is unpredictable. In the work of Meadows et al. [25], the uptake rate constants of polychlorinated biphenyls (PCBs) by triolein-SPMD were 1-2 times of those by brown trout. Their and our results showed that trioleinSPMD concentrates hydrophobic organochlorinated compounds more quickly than fish do.

As regards triolein-SPMD, $R_{\mathrm{s}}$ depends not only on the absorption of targets in triolein, but also on the ability of a chemical to be absorbed by the polyethylene membrane. $R_{\mathrm{s}}$ could be lipid normalized as well. As shown in Table 2, when lipid-normalized, the rate constants for fish were larger than those for trioleinSPMD. The accumulation of a chemical in trioleinSPMD could be an integration of its accumulation in membrane (polyethylene in this case) and in triolein. Both processes are related to the lipophilicity of the compound. In the work of Gale [26], three-compartment model was used for evaluating contaminant accumulation by triolein-SPMD. The model predicts aqueous film control for compounds with moderate to high $K_{\text {ow }}$, rather than polymer film control. Although accumulation in the triolein may be subjected to polymer film control, the overall accumulation in triolein-SPMD should remain under aqueous film control, the lipidnormalized rate constant is therefore much smaller. The diffusivity of hydrophobic chemicals through biomembrane-like polymeric membrane is also controlled by physicochemical properties of the diffusant molecules. The hydrophobic pesticides used in the present study have similar functional groups, size and steric effects and they are most likely the major factors controlling diffusion rates in the membrane. Since uptake behaviors for different pesticides, when compared on the basis of wet weight, were similar for triolein-SPMD and for rainbow trout, it appears that polyethylene membrane may serve as a good model of biological membrane with respect to the uptake of truly dissolved hydrophobic chemicals.

Previous work has shown that triolein-SPMD could accumulate organic pollutants to the levels similar to or even higher than those in aquatic organisms. For example, after $28 \mathrm{~d}$ of exposure of triolein-SPMD adjacent to caged channel fish in the water at the mouths of five Lake Michigan tributaries, they accumulated a greater number of chemicals and at higher concentrations than the fish [17]. However in the work of Prest et al. [16], levels of sequestered pesticides and PCBs were approximately 1.6 times higher in freshwater clams (Corbicula fluminea) than those in triolein-SPMD after 2 months of exposure in the river water $\left(10^{\circ} \mathrm{C}\right)$. Moreover, another result showed that blue mussels were more efficient at sequestering polycyclic aromatic hydrocarbons than were SPMDs [4]. The lipid content in mussels (Anodonta piscinalis) is about 10 times that in clam [22]. It has been demonstrated that for organochlorinated pesticides whose molecules are often characterized by relatively high bond rotational and conformational freedom, each $5^{\circ} \mathrm{C}$ increase in water temperature results in 1.3-2-fold increase in uptake rates. Therefore, both types of organism and temperature could affect the uptake kinetics of pesticides by triolein-SPMD or by aquatic organisms.

\subsection{Comparison of pesticides uptake by triolein-SPMDS and rainbow trout}

Generally in steady state, the accumulation of hydrophobic organic compounds, such as pesticides, by aquatic organisms could be predicted through the 


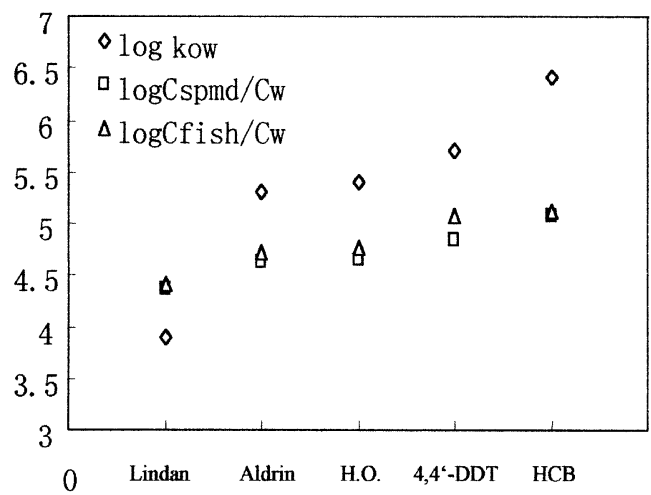

Fig. 2. Relationship between $\log K_{\mathrm{ow}}$ values and the concentration factors of rainbow trout and SPMD.

linear relationship between $\mathrm{BCF}$ (or BAF) and $K_{\text {ow }}$ [27,28]. For compound whose $\log K_{\text {ow }}$ varies from 5 to 7 , the prediction according to linear relationship between $\log$ BCF and $\log K_{\text {ow }}$ could result in a better fit. However for compounds whose $\log K_{\text {ow }}<5$, the prediction would be affected by its strong tendency to remain in the water phase and biodegradation in biota. For a compound whose $\log K_{\text {ow }}>7$, its association with organic matter would make it less bioavailable to biota [29]. Although equilibrium was not reached for most pesticides under 21-d exposure period, correlations between $C_{\mathrm{L}} / C_{\mathrm{w}}$ and $K_{\text {ow }}\left(R^{2}=0.887\right)$ and $C_{\mathrm{f}} / C_{\mathrm{w}}$ and $K_{\text {ow }}\left(R^{2}=0.931\right)$ could be observed in Fig 2, where $\log C_{\mathrm{L}} / C_{\mathrm{w}}$ or $\log C_{\mathrm{f}} / C_{\mathrm{w}}$ was plotted versus $\log K_{\mathrm{ow}}$. This figure was a clear indication that triolein-SPMD accumulates chlorinated pesticides in a quite similar way as fish do and accumulations of individual pesticide either by triolein-SPMD or by fish could be predicted by its $\log K_{\text {ow }}$.

Biomonitoring of aquatic organisms has been used as supplementary to monitoring aqueous concentration, because it provides not only information on environmental pollution, but also the potential ecological risks of an anthropogenic chemicals. However, the use of aquatic organisms in biomonitoring work involves certain problems, such as individual and species variability by sex, age, and physiological state of the organism. All aquatic species are confined to their geographic distribution areas, which makes it difficult to compare data from locations not covered by the distribution area of a single species. In addition, very few, if any, organisms may survive harsh environmental conditions such as deposits of highly contaminated and toxic sediments or industrial effluents. The significant similarity between bioconcentration by rainbow trout and biomimetic concentration by triolein-SPMD for chlorinated pesticides at concentration level close to that observed in natural environment confirms the applicability of the device as surrogate for biomonitoring work. In addition, monitoring with triolein-SPMD is less complex and cost-effective than those using aquatic organisms.

\section{Conclusion}

In the laboratory continuous-flow system and at a concentration level close to that in natural environment, the uptake kinetics of five representative chlorinated pesticides by triolein-SPMD and by rainbow trout were quite similar. The time-dependent accumulation of the pesticides in triolein-SPMD or in fish was linear and only lindan could reach a steady state in fish when exposed for $21 \mathrm{~d}$. However after 21-d exposure, there were good correlations between $\log C_{\mathrm{f}} / C_{\mathrm{w}}$ or $\log C_{\mathrm{L}} / C_{\mathrm{w}}$ and $\log K_{\text {ow }}$, indicating the accumulation process was controlled by the lipophilicity of the chemical. Our results provide additional information on the applicability of triolein-SPMD as a standard testing device for biomonitoring work.

\section{Acknowledgements}

This work was supported by Chinese Academy of Sciences (KZCX2-410) and Natural Science Foundation of China (29977024).

\section{References}

[1] Sodergren A. Monitoring of persistent, lipophilic pollutants in water and sediment by solvent-filled dialysis membranes. Ecotoxicol Environ Saf 1990;19:143-9.

[2] Huckins JN, Tubergen MW, Manuweera GK. Semipermeable membrane devices containing model lipid: a new approach to monitoring the bioavailability of lipophilic contaminants and estimating their bioconcentration potential. Chemosphere 1990;20:533-52.

[3] Huckins J, Petty J, Lebo J, Orazio C. Comment on accumulation of organochlorine pesticides and PCBs by semipermeable membrane devices and Mytilus edulis in New Bedford Harbor. Environ Sci Technol 1997;31: 3732-3.

[4] Axelman J, Naes K, Naf C, Broman D. Accumulation of polycyclic aromatic hydrocarbons in semipermeable membrane devices and caged mussels (Mytilus edulis) in relation to water column phase distribution. Environ Toxicol Chem 1999;18:2454-61.

[5] Bergqvist P-A, Srandberg B, Ekelund R, Rappe C, Granmo A. Temporal monitoring of organochlorine compounds in seawater by semipermeable membranes following a flooding episode in Western Europe. Environ Sci Technol 1998;32:3887-92.

[6] Lebo JA, Gale RW, Petty JD, Tillitt DE, Huckins JN, Meadows JC, Orazio CE, Echols KR, Schroeder DJ, Inmon LE. Use of semipermeable membrane device as an 
in situ sampler of waterborne bioavailable PCDD and PCDF residues at sub-parts-per-quadrillion concentrations. Environ Sci Technol 1995;29:2886-92.

[7] Petty JD, Poulton BC, Charbonneau CS, Huckins JN, Jones SB, Cameron JT, Prest HF. Determination of bioavailable contaminants in the lower Missouri River following the flood of 1993. Environ Sci Technol 1998; 32:837-42.

[8] Zimmerman LR, Thurman EM, Bastian KC. Detection of persistent organic pollutants in the Mississippi Delta using semipermeable membrane devices. Sci Total Environ 2000;248:169-79.

[9] Ockenden WA, Sweetman AJ, Prest HF, Steinnes E, Jones $\mathrm{KC}$. Toward an understanding of the global atmospheric distribution of persistent organic pollutants: the use of semipermeable membrane devices as time-integrated passive samplers. Environ Sci Technol 1998;32:2795-803.

[10] Lohmann B, Corrigan BP, Howsam M, Jones KC, Ockenden WA. Further developments in the use of semipremeable membrane devices (SPMDs) as passive air samplers for persistent organic pollutants: field application in a spatial survey of PCDD/Fs and PAHS. Environ Sci Technol 2001;35:2576-82.

[11] Wang Y, Wang Z, Ma M, Wang CX, Mo Z. Monitoring priority pollutants in a sewage treatment process by dichloromethane extraction, triolein-semipermeable membrane device (SPMD). Chemosphere 2001;43:339-46.

[12] Granmo A, Ekelund R, Berggren M, Brorstrom-Lunden E, Berbgqvist P. Temporal trend of organochlorine marine pollution indicated by concentrations in mussels, semipermeable membrane devices, and sediment. Environ Sci Technol 2000;34:3323-9.

[13] StuerLauridsen F, Kjolholt J. Identification of selected hydrophobic organic contaminants in wastewater with semipermeable membrane devices (SPMDs). Water Res 2000;34:3478-82.

[14] Gustavson KE, Harkin JM. Comparison of sampling techniques and evaluation of semipermeable membrane devices (SPMDs) for monitoring polynuclear aromatic hydrocarbons (PAHs) in groundwater. Environ Sci Technol 2000;34:4445-51.

[15] Huckins JN, Petty JD, Lebo JA, Orazio CE, Prest HF, Tillitt DE, Ellis GS, Johnson BT, Manuweera GK. Semipermeable membrane devices (SPMDs) for the concentration and assessment of bioavailable organic contaminants in aquatic environments. In: Toxicology G, Ostrander O, editors. Book chapter in techniques in aquatic. Boca Raton, FL: CRC-Lewis Publishers, 1996. p. $625-55$.

[16] Prest HF, Jarman WM, Burns SA, Weismuller TM, Huckins JN. Passive water sampling via semipermeable membrane devices in concert with bivalves in the Sacramenta/San Joaquin River Delta. Chemosphere 1992; 25:1811-23.

[17] Wood CA. Comparison of chemical bioaccumulation by lipid-filled semipermeable membrane devices (SPMDS) and Caged channel catfish exposed to select lake Michigan tributaries. Second International Semipermeable Membrane Devices Workshop, US Department of the Interior. Columbia Environmental Research Center, Columbia, MO, 1994.

[18] Devita W.M, Crunkilton R.L. Effectiveness of semipermeable membrane devices, fathead minnows, and rusty crayfish to monitor polynuclear aromatic hydrocarbons in an urban stream. Second International Semipermeable Membrane Devices Workshop, US Department of the Interior, Columbia Environmental Research Center, Columbia, MO, 1994.

[19] Herve S, Prest HF, Heinonen P, Hyotylainen T, Koistinen J, Paasivirta J. Lipid-filled semipermeable membrane devices and mussels as samplers of organochlorine compounds in lake water. Environ Sci Pollut Res 1995; 2:24-30.

[20] Peven CS, Uhler AD, Querzoli FJ. Caged mussels and semipermeable membrane devices as indicators of organic contaminant uptake in Dorchester and Duxbury Bays, Massachusetts. Environ Toxicol Chem 1996;15:144-9.

[21] Hofelt CS, Shea D. Accumulation of organochlorine pesticides and PCBs by semipermeable membrane devices and Mytilus edulis in New Bedford Harbor. Environ Sci Technol 1997;31:154-9.

[22] Strandberg B. The use of Semipermeable membrane devices in studies of concentrations, distribution and fate of organochlorine compounds in the environment. Ph.D. thesis, Umeo̊, Sweden, 1998.

[23] Bergqvist P-A, Strandberg B, Rappe C. Lipid removal using semipermeable membranes (SPMDs) in PCDD and PCDF analysis of fatrich environmental samples. Chemosphere 1998;38:933-43.

[24] OCED guideline for testing of chemicals, part of Bioconcentration: Flow-Through Fish Test, adopted at 12 May 1981.

[25] Meadows JC, Echols KR, Huckins JN, Borsuk FA, Carline RF, Tillitt DE. Estimation of uptake rate constants for PCB congeners accumulated by semipermeable membrane devices and brown trout (Salmo trutta). Environ Sci Technol 1998;32:1847-52.

[26] Gale RW. Three-compartment model for contaminant accumulation by semipermeable membrane devices. Environ Sci Technol 1998;32:2292-300.

[27] Di Toro DM, Zarba CS, Hansen DJ, Berry WJ, Swartz RC, Cowan CE, Pavlou SP, Allen HE, Thomas NA, Paquin PR. Technical basis for establishing sediment quality criteria for nonionic organic chemicals using equilibrium partitioning. Environ Toxicol Chem 1991; 10:1541-83.

[28] Verhaar HJM, Jongh JJ, Hermens JM. Modeling the bioconcentration of organic compounds by fish: a novel approach. Environ Sci Technol 1999;33:4069-72.

[29] Norstrom RJ, Muie D. Chlorinated hydrocarbon contaminants in arctic marine mammals. Sci Total Environ 1994;154:107-28. 